\title{
Synthesis of 2-amino-1,4-benzodiazepin-5-ones from 2-nitrobenzoic acid and $\alpha$-aminonitriles
}

\author{
Eyad A. Younes ${ }^{a}$, Ahmad Q. Hussein ${ }^{a *}$, Mitchell A. May ${ }^{b}$, and Frank R. Fronczek ${ }^{b}$ \\ ${ }^{a}$ Chemistry Department, University of Jordan, Amman, Jordan \\ ${ }^{b}$ Department of Chemistry, Louisiana State University, Baton Rouge, LA 70803, USA
}

E-mail: aqhussein@yahoo.com

\begin{abstract}
Condensation of $\alpha$-aminonitriles with 2-nitrobenzoyl chloride gives the corresponding Schiff bases, which furnish, upon reduction with $\mathrm{Zn}$, the corresponding 2-amino-3,4-dihydro-5H-1,4benzodiazepin-5-ones. However, reduction of the Schiff base obtained from the condensation of 1-aminocyclohexanecarbonitrile with 2-nitrobenzaldehyde gives the methanol adduct of 2aminobenzaldehyde trimer.
\end{abstract}

Keywords: 2-Nitrobenzoic acid, 2-nitrobenzaldehyde, 2-aminobenzaldehyde, 2-amino-1,4benzodiazepine, $\alpha$-aminonitrile, tribenzo $[b, f, j][1.5 .9]$ triazacyclododecene, crystal structure

\section{Introduction}

The 1,4-benzodiazepine scaffold imparts biological activity to molecules which incorporate this heterocyclic nucleus, giving molecules capable of binding to brain receptors, and enhancing the effect of the neurotransmitter $\gamma$-aminbutyric acid. ${ }^{1}$ Consequently, many derivatives of $1,4-$ benzodiazepines display a wide range of biological activities and constitute an essential part of the structure of a significant number of pharmacologically active compounds which exhibit anxiolytic, anticonvulsant, hypnotic, sedative, and muscle relaxant effects. ${ }^{2}$ Some naturallyoccurring 1,4-benzodiazepines exhibit antitumour and antibiotic activities. ${ }^{1 \mathrm{~d}, 3}$ Since the introduction of chlorodiazepoxide (Librium) and diazepam (Valium) to the market by Hoffmann La-Roche in the early 1960s, over a dozen benzodiazepines are now in medicinal use. ${ }^{4}$

The search for new 1,4-benzodiazepines is still an active area for synthetic chemists, and there is an increasing demand for structural diversification through the development of new synthetic protocols, ${ }^{2 \mathrm{a}, 5}$ whereby structural modifications and new substituents can be introduced into the basic skeleton. ${ }^{6}$ In this context, we report here the synthesis of 2-amino-3,4-dihydro-5H1,4-benzodiazepin-5-ones starting with 2 -nitrobenzoic acid and $\alpha$-aminonitriles. 


\section{Results and Discussion}

The condensation of 2-nitrobenzoyl chloride $\mathbf{1}$ with $\alpha$-aminonitriles $\mathbf{2}$ was found to proceed well in THF at $0{ }^{\circ} \mathrm{C}$ in the presence of triethylamine as a base, to furnish the corresponding amides $\mathbf{3}$ as yellow crystalline solids in high yields (Scheme 1).

The structures of these compounds were elucidated through their IR, NMR, and HRMS spectra. In the IR spectra, these compounds exhibited an absorption at about $2240 \mathrm{~cm}^{-1}$, characteristic of the $\mathrm{C} \equiv \mathrm{N}$ stretching mode, in addition to a strong absorption at about $1640 \mathrm{~cm}^{-1}$ and a broad absorption at 3220-3245 $\mathrm{cm}^{-1}$, assigned, respectively, to the $\mathrm{C}=\mathrm{O}$ and $\mathrm{N}-\mathrm{H}$ bond stretching of the amide linkage. The HRMS spectra revealed the correct molecular formulas through exact mass measurements of either the $[\mathrm{M}-\mathrm{H}]^{-}$ion, in the negative ion mode, or the $[\mathrm{M}+\mathrm{Na}]^{+}$ion, in the positive ion mode, with less than $\pm 1 \mathrm{ppm}$ error. The ${ }^{1} \mathrm{H}-$ and ${ }^{13} \mathrm{C}-\mathrm{NMR}$ spectra were consistent with the assigned structures; the $\mathrm{C} \equiv \mathrm{N}$ carbon appeared at 116-120 ppm, and the assignment of the remaining carbon and proton signals in each case were straightforward.

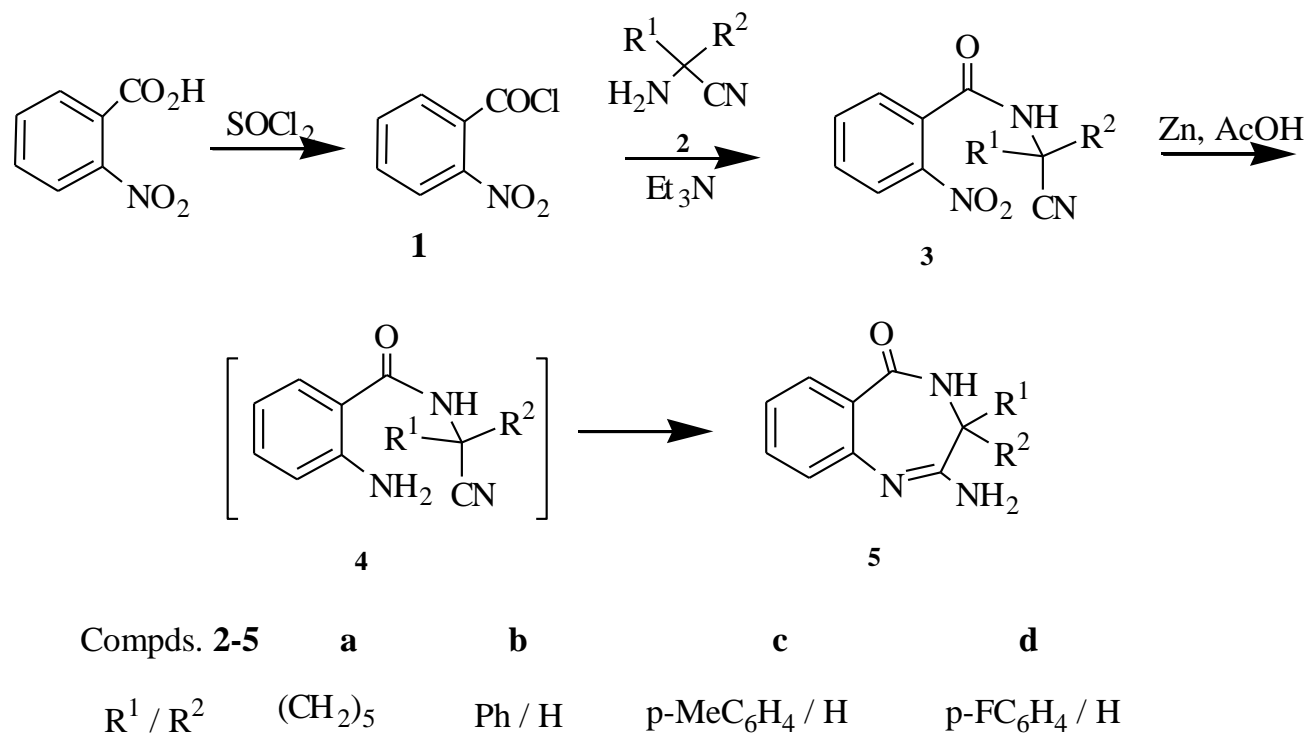

Scheme 1. Synthetic pathway for 2-amino-1,4-benzodiazepin-5-ones.

Reduction of the nitro group in these compounds to an amino group, was achieved by zinc powder with acetic acid in dichloromethane at room temperature. Such mild reaction conditions were intentionally employed with the aim of isolating intermediate 2-aminobenzamide derivatives 4 , however the reactions proceeded further to furnish the cyclization products, namely the corresponding 2,4-benzodiazepinones $\mathbf{5}$. That cyclization of the intermediates $\mathbf{3}$ had occurred was evident from the absence of a $\mathrm{C} \equiv \mathrm{N}$ stretching in the IR spectra of the isolated products, confirming the involvement of the nitrile functionality in the cyclization process. The IR spectra also revealed $\mathrm{C}=\mathrm{N}$ stretching at about $1590-1615 \mathrm{~cm}^{-1}$ and $\mathrm{NH}_{2}$ stretching modes in 
the range $3240-3290$ and $3360-3380 \mathrm{~cm}^{-1}$ in addition to absorption bands at about 1630 and at $3450-3480 \mathrm{~cm}^{-1}$ assigned to the amide functionality. A downfield shift was noted for the ${ }^{13} \mathrm{C}$ NMR signal of the original $\mathrm{C} \equiv \mathrm{N}$ carbon from 116-120 ppm in the acyclic 2-nitrobenzamides 3 to 149-150 ppm for the C-2 carbon in compounds $\mathbf{5}$, being flanked by two nitrogen atoms in the cyclic structure. The C-3 proton in compounds $\mathbf{5 b}-\mathbf{d}$ appeared as a doublet at $6.2-6.4 \mathrm{ppm}(1 \mathrm{H}$, $J=7.5 \mathrm{~Hz}$ ). The HRMS spectra revealed the correct molecular formulas through exact mass measurements of the $[\mathrm{M}+\mathrm{Na}]^{+}$ion, in the positive ion mode, with $\leq \pm 1 \mathrm{ppm}$ error.

In an attempt to develop an analogous synthetic route for the 2-amino-3H-1,4benzodiazepine 8 through the condensation of $\alpha$-aminonitrile 2a with 2-nitrobenzaldehyde was investigated (Scheme 2). The corresponding Schiff base $\mathbf{6}$ was obtained in fairly high yield, the structure of which was elucidated through spectral data. Thus, the ${ }^{1} \mathrm{H}-\mathrm{NMR}$ spectrum of compound 6 revealed, in addition to multiplets at 7.60-8.07 $(4 \mathrm{H})$ and $1.67-1.99 \mathrm{ppm}(10 \mathrm{H})$, assigned, respectively, to the aromatic and the cyclohexyl protons, a signal at $9.07(1 \mathrm{H})$ for the methine proton. The IR spectrum showed an absorption due to $\mathrm{C} \equiv \mathrm{N}$ bond stretching at $2220 \mathrm{~cm}^{-1}$.

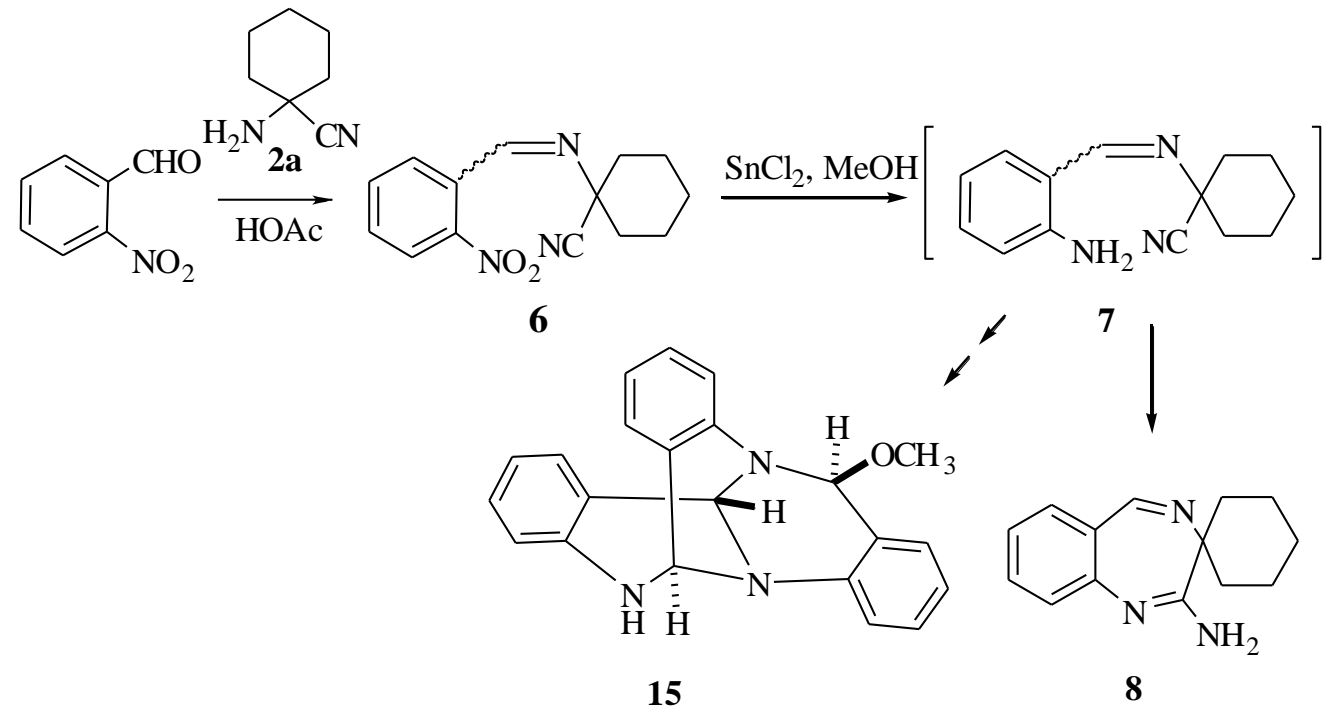

Scheme 2. Reduction of Schiff base 6.

Attempted transformation of this compound into the corresponding 2-amino derivative 7, and thence to the cyclization product $\mathbf{8}$ derived therefrom, through reduction with $\mathrm{SnCl}_{2} /$ methanol, gave a product with spectral data that were difficult to interpret. The ${ }^{1} \mathrm{H}-\mathrm{NMR}$ spectrum of the revealed, in addition to signals in the aromatic region $(13 \mathrm{H})$, a singlet $(3 \mathrm{H})$ at 3.95 and three additional singlets $(1 \mathrm{H}$ each) at $5.09,5.30$, and $5.53 \mathrm{ppm}$. The IR spectrum exhibited a broad absorption at about $3370 \mathrm{~cm}^{-1}$, and HRMS gave an exact molar mass of 342.16009. To confirm the structure of this compound, an X-ray structure determination was undertaken, which proved that the compound is the methanol adduct 15 (Figure 1) of a 2- 
aminobenzaldehyde trimer. Analogous trimers, as well as tetramers, of 2-aminobenzaldehyde have been previously reported, formed through the action of complexing metal ions on 2aminobenzaldehyde. ${ }^{7}$ However, only a tentative reaction mechanism had been suggested for such condensation reactions, whereby a dimeric Schiff base intermediate, formed by self-condensation of two molecules of the 2-aminobenzaldehyde, was assumed. ${ }^{8}$

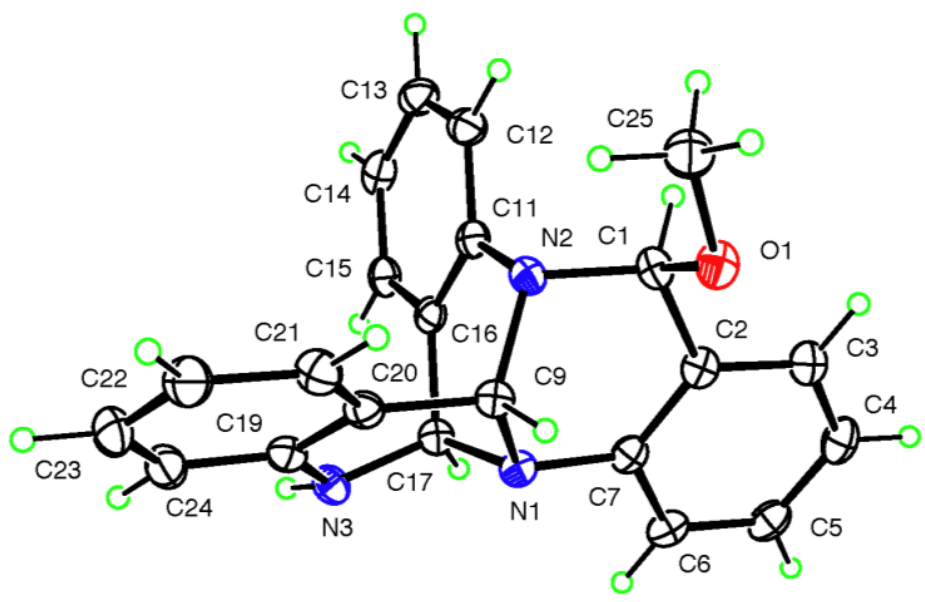

Figure 1. X-ray structure of compound 15. One of two independent molecules in the asymmetric unit is shown.

We could not, however, find any trace of trimer formation when 2-nitrobenzaldehyde was subjected to reduction under the same reaction conditions employed for the above-mentioned formation of 15. This excludes the intermediacy of 2-nitrobenzaldehyde, which might have been formed through hydrolysis of the Schiff base 6, in the formation of the trimer in the present study. Therefore, we suggest that first, a metal complex is probably formed between the aminonitrile Schiff base 7 and $\mathrm{Sn}^{2+}$ ion, rendering the imine carbon electrophilic enough to initiate subsequent reaction with a second uncomplexed 2-aminobenzylidene Schiff base molecule 7 to give the dimeric structure 9, which undergoes elimination of an aminonitrile molecule (arrows on 9, Scheme 3). The resulting product $\mathbf{1 0}$ subsequently adds to a third Schiff base giving 11 which, after loss of an aminonitrile molecule (arrows on 11) produces compound 12. Intramolecular amine-to-imine addition (arrows on 12) generates 13 and loss of the third equivalent of aminonitrile yields the 2-aminobenzaldehyde trimer, tribenzo[b,f,j][1.5.9]triazacyclododecene 14. Finally, this trimer adds a molecule of methanol (solvent), which triggers two intramolecular cyclizations (arrows on 14) involving the nucleophilic nitrogen atoms and the electrophilic imine groups to give the final tricyclic product 15. 


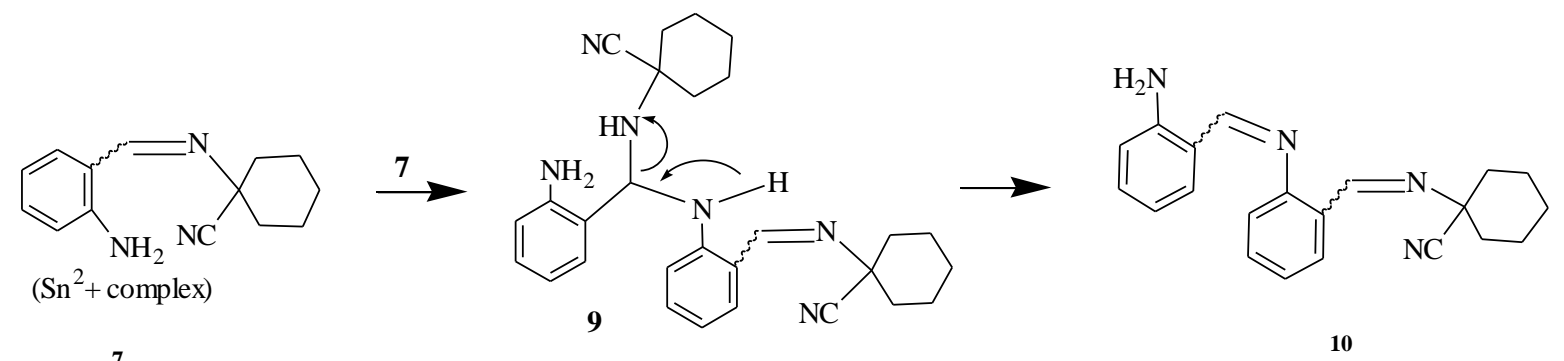<smiles>CC(C)(C)NC1(c2ccccc2N=Cc2ccccc2N)CCN(c2ccccc2C=NC2(C#N)CCCCC2)CC1(C#N)C(C)(C)C</smiles>

11

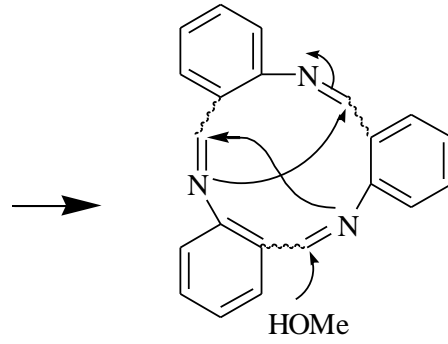

14

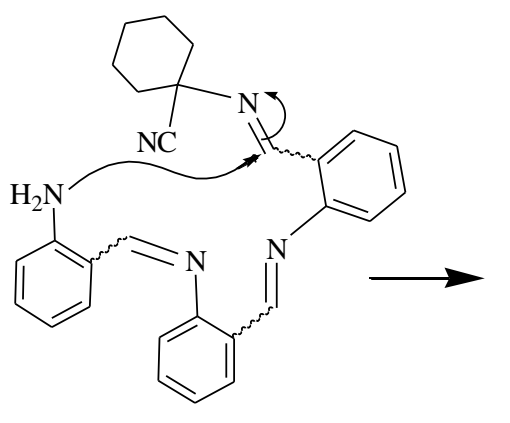

12

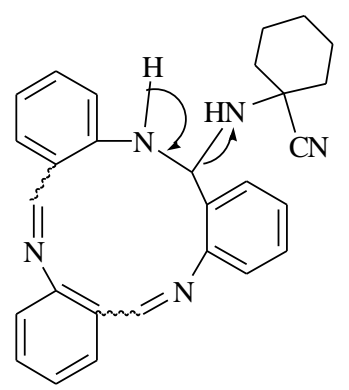

13

Scheme 3. Suggested reaction mechanism for the formation of trimer 15.

\section{Experimental Section}

General. All solvents and chemicals were of commercial grade (Aldrich), and were used without further purification. Melting points were measured on a Harris melting point apparatus and are uncorrected. ${ }^{1} \mathrm{H}-\mathrm{NMR}$ and ${ }^{13} \mathrm{C}-\mathrm{NMR}$ spectra were measured at room temperature on a Bruker DPX-300 instrument, operating at 300 and $75 \mathrm{MHz}$, respectively, using tetramethylsilane $(0.00$ $\mathrm{ppm})$ as internal standard. IR spectra were recorded, as potassium bromide $(\mathrm{KBr})$ discs, on a Nicolet Impact-400 FT-IR spectrophotometer. High resolution mass spectra (HRMS) were measured in positive and/or negative ion mode by electrospray ionization (ESI) on a Bruker Apex IV instrument at 7.0 Tesla; samples were dissolved in acetonitrile, diluted in spray solution $(0.1 \%$ formic acid in 1:1 v/v methanol-water), and infused using a syringe pump at a flow rate of $2 \mu \mathrm{L} / \mathrm{min}$, using Arginine cluster for external calibration in the mass range $\mathrm{m} / \mathrm{z}$ 175-871. 


\section{Preparation of compounds (3a-d). General procedure}

2-Nitrobenzoyl chloride $(10.0 \mathrm{mmol})$ was added to a solution of the aminonitrile, prepared according to a literature procedure, ${ }^{9}(11.0 \mathrm{mmol})$ in dry THF $(50 \mathrm{~mL})$ containing $\mathrm{Et}_{3} \mathrm{~N}(12.0$ $\mathrm{mmol}$ ) at $0{ }^{\circ} \mathrm{C}$. The resulting mixture was stirred for 30 minutes at $\mathrm{rt}$, the insoluble salt was filtered off, and the solvent was evaporated under reduced pressure. The remaining solid was collected and recrystallized from $\mathrm{MeOH} / \mathrm{H}_{2} \mathrm{O}$.

$N$-(1-Cyanocyclohexyl)-2-nitrobenzamide (3a). Yield 94\%, yellow solid, m.p. 152-154 ${ }^{\circ} \mathrm{C}$. IR $(\mathrm{KBr}) v$ 3216, 2239, 1656, 1639, 1523, $1344 \mathrm{~cm}^{-1}$. HRMS (ESI): $\mathrm{m} / \mathrm{z}$ calculated for [M-H] 272.1041, found 272.1041. ${ }^{1} \mathrm{H}-\mathrm{NMR}\left(300 \mathrm{MHz}, \mathrm{CDCl}_{3}\right) \delta 1.25-2.42(\mathrm{~m}, 10 \mathrm{H}), 6.07$ (brs, $\left.1 \mathrm{H}\right)$, 7.49-7.60 (m, 2H), 7.65-7.71(m, 1H), $8.04(\mathrm{~d}, J=9.8 \mathrm{~Hz}, 1 \mathrm{H}) .{ }^{13} \mathrm{C}-\mathrm{NMR}\left(75 \mathrm{MHz}, \mathrm{CDCl}_{3}\right) \delta$ 22.1, 24.6, 35.0, 52.4, 119.2, 124.5, 128.9, 130.8, 131.8, 134.0, 145.9, 165.6.

$N$-(Cyano(phenyl)methyl)-2-nitrobenzamide (3b). Yield 74\%, yellow solid, m.p. 155-156 ${ }^{\circ} \mathrm{C}$. IR $(\mathrm{KBr}) v 3246,3036,2243,1649,1525,1347 \mathrm{~cm}^{-1}$. HRMS (ESI) $\mathrm{m} / z$ calculated for $[\mathrm{M}+\mathrm{Na}]^{+}$ 304.0693, found 304.0693. ${ }^{1} \mathrm{H}-\mathrm{NMR}\left(300 \mathrm{MHz}, \mathrm{CDCl}_{3}\right) \delta 6.26(\mathrm{~d}, \mathrm{~J}=8.2 \mathrm{~Hz}, 1 \mathrm{H}), 6.70$ (brs, $\left.1 \mathrm{H}\right)$, 7.26-7.68 (m, 8H), $8.05(\mathrm{~d}, \mathrm{~J}=8.0 \mathrm{~Hz}, 1 \mathrm{H}) .{ }^{13} \mathrm{C}-\mathrm{NMR}\left(75 \mathrm{MHz}, \mathrm{CDCl}_{3}\right) \delta 44.6,116.8,124.7$, 127.2, 128.7, 129.4, 129.7, 132.3, 131.1, 133.9, 134.1, 146.0, 165.6.

$N$-(Cyano-(p-tolyl)methyl)-2-nitrobenzamide (3c). Yield 77\%, yellow solid, m.p. 180-182 ${ }^{\circ} \mathrm{C}$. IR $(\mathrm{KBr}) v$ 3237, 2244, 1648, 1530, $1349 \mathrm{~cm}^{-1}$. HRMS (ESI) $\mathrm{m} / z$ calculated for $[\mathrm{M}+\mathrm{Na}]^{+}$ 318.0849, found 318.0849. ${ }^{1} \mathrm{H}-\mathrm{NMR}\left(300 \mathrm{MHz} \mathrm{CDCl}_{3}\right) \delta 2.34(\mathrm{~s}, 3 \mathrm{H}), 6.23(\mathrm{~d}, J=8.1 \mathrm{~Hz}, 1 \mathrm{H})$, 6.67 (brs, $1 \mathrm{H}), 7.22-7.72(\mathrm{~m}, 7 \mathrm{H}), 8.08(\mathrm{~d}, J=9.2 \mathrm{~Hz}, 1 \mathrm{H}) .{ }^{13} \mathrm{C}-\mathrm{NMR}\left(75 \mathrm{MHz}, \mathrm{CDCl}_{3}\right) \delta 21.2$, 44.4, 117.0, 124.7, 127.1, 128.7 , 129.4, 130.1, 131.1, 131.2, 139.9, 146.0, 165.7.

N-(Cyano-(4-fluorophenyl)methyl)-2-nitrobenzamide (3d). Yield 78\%, yellow solid, m.p. 127-128 ${ }^{\circ} \mathrm{C}$. IR (KBr) v 3255, 2957, 2917, 1524, $1347 \mathrm{~cm}^{-1}$. HRMS (ESI) $\mathrm{m} / z$ calculated for $[\mathrm{M}+\mathrm{Na}]^{+}$322.0598, found 322.0598. ${ }^{1} \mathrm{H}-\mathrm{NMR}\left(300 \mathrm{MHz}, \mathrm{CDCl}_{3}\right) \delta 6.35(\mathrm{~d}, J=8.3 \mathrm{~Hz}, 1 \mathrm{H}), 6.43$ (brs, $1 \mathrm{H}), 7.13-7.19(\mathrm{~m}, 2 \mathrm{H}), 7.56-87.77(\mathrm{~m}, 5 \mathrm{H}), 8.14(\mathrm{~d}, J=8.0 \mathrm{~Hz}, 1 \mathrm{H}) .{ }^{13} \mathrm{C}-\mathrm{NMR}(75 \mathrm{MHz}$, $\left.\mathrm{CDCl}_{3}\right) \delta 44.0,116.4,116.7,116.8,124.9,128.3$,128.7, 129.2, 129.3, 131.3, 134.1, 165.6.

\section{Preparation of compounds (5a-d). General procedure}

The nitro compound 3a-c $(3.0 \mathrm{mmol})$ and zinc powder $(1.00 \mathrm{~g})$ were suspended in $\mathrm{CH}_{2} \mathrm{Cl}_{2}(30$ $\mathrm{mL}$ ) in a double-necked flask equipped with a condenser. To the stirred mixture was added dropwise within $5 \mathrm{~min}$ AcOH $(2 \mathrm{~mL})$ dissolved in $\mathrm{CH}_{2} \mathrm{Cl}_{2}(10 \mathrm{~mL})$. Stirring was continued for 1 h. Excess zinc powder was filtered off and the filtrate was washed with saturated $\mathrm{NaHCO}_{3}$ solution ( 3 x $15 \mathrm{~mL}$ ), dried over $\mathrm{MgSO}_{4}$, and the solvent was evaporated under reduced pressure. The product was collected and recrystallized from $\mathrm{MeOH}$.

2-Aminospiro[1,4-benzodiazepine-3,1'-cyclohexane]-5(4H)-one (5a). Yield 85\%, white solid, m.p. $149-150{ }^{\circ} \mathrm{C}$. IR (KBr): $v$ 3479, 3377, 3294, 1632, $1616 \mathrm{~cm}^{-1}$. HRMS (ESI): $\mathrm{m} / z$ calculated for $[\mathrm{M}+\mathrm{Na}]^{+}$266.1264, found 266.1264. ${ }^{1} \mathrm{H}-\mathrm{NMR}\left(300 \mathrm{MHz}, \mathrm{CDCl}_{3}\right): \delta 1.25-2.42(\mathrm{~m}, 10 \mathrm{H})$, 5.59 (brs, 2H), 6.06 (brs, $1 \mathrm{H}), 6.60-6.38$ (m, 2H), 7.19-7.30 (m, 2H). ${ }^{13} \mathrm{C}-\mathrm{NMR}(75 \mathrm{MHz}$, $\left.\mathrm{CDCl}_{3}\right): \delta 22.1,24.6,35.6,51.6,114.2,116.5,117.6,119.9,127.2,133.1,149.3,168.5$. 
2-Amino-3-phenyl-3,4-dihydro-1,4-benzodiazepin-5-one (5b). Yield 83\%, white solid, m.p. 168-169 ${ }^{\circ} \mathrm{C}$. IR (KBr): $v$ 3487, 3377, 3252, 1629, $1583 \mathrm{~cm}^{-1}$. HRMS (ESI): $\mathrm{m} / \mathrm{z}$ calculated for $[\mathrm{M}+\mathrm{Na}]^{+}$274.0950, found 274.0951. ${ }^{1} \mathrm{H}-\mathrm{NMR}\left(300 \mathrm{MHz}, \mathrm{CDCl}_{3}\right): \delta 5.61$ (brs, 2H), $6.26(\mathrm{~d}$, $J=8.1 \mathrm{~Hz}, 1 \mathrm{H}), 6.56$ (brs, $1 \mathrm{H}), 6.60(\mathrm{~m}, 2 \mathrm{H}), 7.24-7.31(\mathrm{~m}, 1 \mathrm{H}), 7.47-7.57(\mathrm{~m}, 6 \mathrm{H}) .{ }^{13} \mathrm{C}-\mathrm{NMR}(75$ $\mathrm{MHz}_{\mathrm{CDCl}}$ ): $\delta$ 44.3, 113.4, 116.7, 117.5, 117.6, 127.0 , 127.2, 129.5, 133.4, 149.5, 168.1 .

2-Amino-3,4-dihydro-3-p-tolyl-1,4-benzodiazepin-5-one (5c). Yield 70\%, white solid, m.p. 171-172 ${ }^{\circ} \mathrm{C}$. IR (KBr): $v$ 3452, 3362, 3235, $1637 \mathrm{~cm}^{-1}$. HRMS (ESI): $\mathrm{m} / z$ calculated for $[\mathrm{M}+\mathrm{Na}]^{+}$ 288.1107, found 288.1107. ${ }^{1} \mathrm{H}-\mathrm{NMR}\left(300 \mathrm{MHz}, \mathrm{CDCl}_{3}\right.$ ): $\delta 2.36$ (s, 3H), 5.62 (brs, 2H), 6.18 (d, $J=7.3 \mathrm{~Hz}, 1 \mathrm{H}), 6.58-6.70(\mathrm{~m}, 2 \mathrm{H}), 7.210-7.43(\mathrm{~m}, 7 \mathrm{H}) .{ }^{13} \mathrm{C}-\mathrm{NMR}\left(75 \mathrm{MHz}, \mathrm{CDCl}_{3}\right): \delta 21.2$, 44.6, 113.3, 116.6, 117.6, 117.8, 127.1, 127.3, 130.1, 130.2, 133.4, 139.7, 149.4, 168.2.

2-Amino-3,4-dihydro-3-p-fluorophenyl-1,4-benzodiazepin-5-one (5d). Yield 58\%, white solid, m.p. $115-116{ }^{\circ} \mathrm{C}$. IR (KBr): $v$ 3452, 3362, 3235, $16371 \mathrm{~cm}^{-1}$. HRMS (ESI): $\mathrm{m} / z$ calculated for $[\mathrm{M}+\mathrm{Na}]^{+}$292.0857, found 292.0857. ${ }^{1} \mathrm{H}-\mathrm{NMR}\left(300 \mathrm{MHz}, \mathrm{CDCl}_{3}\right): \delta 4.78(\mathrm{brs}, 1 \mathrm{H}), 5.63$ (brs, 2H), $6.26(\mathrm{~d}, J=8.1 \mathrm{~Hz}, 1 \mathrm{H}), 6.61-6.72(\mathrm{~m}, 2 \mathrm{H}), 7.11-7.16(\mathrm{~m}, 2 \mathrm{H}), 7.22-7.32(\mathrm{~m}, 2 \mathrm{H})$, 7.50-7.56 (m, 2H). ${ }^{13} \mathrm{C}-\mathrm{NMR}\left(75 \mathrm{MHz}, \mathrm{CDCl}_{3}\right): \delta 43.6,113.0,116.3,116.6,117.6,127.2,129.1$, $129.4,133.5,149.5,161.6,164.9,168.1$.

\section{Preparation of 1-(2-nitrobenzylideneamino)cyclohexanecarbonitrile (6)}

A solution of 2-nitrobenzaldehyde $(5.0 \mathrm{mmol})$ and 1-aminocyclohexanecarbonitrile $(6.0 \mathrm{mmol})$ in $\mathrm{MeOH}$ containing two drops of glacial $\mathrm{AcOH}$ was stirred at $\mathrm{rt}$ for $2 \mathrm{~h}$. The precipitate formed was filtered, washed with $\mathrm{H}_{2} \mathrm{O}$ and recrystallized from EtOH/ $\mathrm{H}_{2} \mathrm{O}$. Yield 70\%, yellow solid, m.p. 53-54 ${ }^{\circ} \mathrm{C}$. IR (KBr): $v$ 2944, 2860, 2222, 1530, $1343 \mathrm{~cm}^{-1}$. HRMS (ESI): $m / z$ calculated for $[\mathrm{M}+\mathrm{Na}]^{+}$280.1056, found 280.1056. ${ }^{1} \mathrm{H}-\mathrm{NMR}\left(300 \mathrm{MHz}, \mathrm{CDCl}_{3}\right): \delta 1.67-1.99(\mathrm{~m}, 10 \mathrm{H}), 7.60$ $(\mathrm{m}, 1 \mathrm{H})$ 7.68-7.73 (m, 1H), 7.93-7.96 (d, 1H), 8.06-8.07 (d, 1H), $9.06(\mathrm{~s}, 1 \mathrm{H}) .{ }^{13} \mathrm{C}-\mathrm{NMR}(75$ $\left.\mathrm{MHz} \mathrm{CDCl}_{3}\right): \delta 22.5,24.5,37.4,65.6,119.7,124.5,130.0,130.5,131.3,133.6,148.8,156.5$.

\section{Preparation of 13-methoxy-7,11b-dihydro-13H-6,12[1',2']-benzeno-6H-quinazolino[3,4-a]- quinazoline (15)}

Compound $6(5.0 \mathrm{mmol})$ ) and $\mathrm{SnCl}_{2} .2 \mathrm{H}_{2} \mathrm{O}(2.00 \mathrm{~g})$ were heated under reflux for $5 \mathrm{~h}$ in $\mathrm{MeOH}$ $(10 \mathrm{~mL})$. The solvent was then evaporated under reduced pressure and the residue was extracted with EtOAc $(20 \mathrm{~mL})$. Saturated aqueous $\mathrm{NaHCO}_{3}(10 \mathrm{~mL})$, and Celite $(2 \mathrm{~g})$ were added, and the mixture was stirred at $\mathrm{rt}$ for $1 \mathrm{~h}$, then filtered. The organic layer was separated and the solvent was evaporated under reduced pressure. The resulting solid was washed with $\mathrm{H}_{2} \mathrm{O}$, and recrystallized from $\mathrm{MeOH}$. Yield 54\%, yellow solid, m.p. 215-217 ${ }^{\circ} \mathrm{C}$. IR (KBr): v 3372, 3287 , 1606, $1479 \mathrm{~cm}^{-1}$. HRMS (ESI): $\mathrm{m} / z$ calculated for $[\mathrm{M}+\mathrm{H}]^{+} 342.1601$, found 342.1601. ${ }^{1} \mathrm{H} \mathrm{NMR}$ $\left(300 \mathrm{MHz}, \mathrm{CDCl}_{3}\right): \delta 3.95(\mathrm{~s}, 3 \mathrm{H}), 5.09(\mathrm{~s}, 1 \mathrm{H}), 5.30(\mathrm{~s}, 1 \mathrm{H}), 5.53(\mathrm{~s}, 1 \mathrm{H}), 6.72-6.74(\mathrm{~d}, 1 \mathrm{H})$, 6.85-7.32 (m, 12H). $\left.{ }^{13} \mathrm{C} \mathrm{NMR} \mathrm{(75} \mathrm{MHz} \mathrm{CDCl}_{3}\right): \delta$ 55.6, 63.3, 69.8, 94.0, 117.5, 120.0, 124.1, 124.2, 124.1, 124.4, 127.1, 128.1, 128.6, 128.8, 129.3, 129.4, 129.6, 129.7, 140.9, 142.5, 145.0. 


\section{X-ray Crystallography}

The crystal structure of $\mathbf{1 5}$ was determined using data collected at $T=90 \mathrm{~K}$ with CuK $\alpha$ radiation $(\lambda=1.54178 \AA)$ on a Bruker APEX-II CCD diffractometer, equipped with an Oxford Cryostream cooler. Crystal data: $\mathrm{C}_{22} \mathrm{H}_{19} \mathrm{~N}_{3} \mathrm{O}, M_{\mathrm{r}}=341.4$, monoclinic space group $\mathrm{P} 21 / \mathrm{c}, a=8.7743(2), b=$ 20.4393(5), $c=18.7698(5) \AA, \beta=93.1880(10)^{\circ}, \mathrm{V}=3360.98(14) \AA^{3}, Z=8, D_{\mathrm{x}}=1.349 \mathrm{~g} \mathrm{~cm}^{-3}$, $\theta_{\max }=68.5^{\circ}, R=0.039$ for 6058 data and 478 refined parameters. $\mathrm{H}$ atoms were visible in difference maps, but were placed in idealized positions, except for $\mathrm{N}-\mathrm{H}$ hydrogen atoms, for which coordinates were refined. Maximum and minimum residual electron densities were 0.44 and -0.20 e $\AA^{-3}$, respectively. CCDC 789475.

\section{Acknowledgements}

The first two authors wish to thank the Deanship of Academic Research, University of Jordan for financial support of this work, which is part of the M.Sc. Thesis of E. A. Younes

\section{References}

1. (a) Da Settimo, F.; Taliani, S.; Trincavelli, M. L.; Montali, M.; Martini, C. Curr. Med. Chem. 2007, 14, 2680. (b) Berezhnoy, D.; Gibbs, T. T.; Farb, D. H. Mol. Pharmacol. 2009, 76, 440. (c) Cipolla, L.; Araujo, A. C.; Airoldi, C.; Bini, D. Anti-Cancer Agents Med. Chem. 2009, 9, 1. (d) Thurston, D. E.; Bose, D. S. Chem. Rev. 1994, 94, 433. (e) Bock, M. G.; DiPardo, R. M.; Evans, B. E.; Rittle, K. E.; Whitter, W. L.; Veber, D. F.; Anderson, P. S.; Freidinger, R. M. J. Med. Chem. 1989, 32, 13.

2. (a) Cobo, J.; Nogueras, M.; Low, J. N.; Rodriguez, R. Tetrahedron Lett. 2008, 49, 7271. (b) Kamal, A.; Shankaraiah, N.; Markandeya, N.; Reddy, K. L.; Reddy, C. S. Tetrahedron Lett. 2008, 49, 1465.

3. (a) Fotso, S.; Zabriskie, T. M.; Proteau, P. J.; Flatt, P. M.; Santosa, D. A.; Sulastri, S.; Mahmud, T., J. Nat. Prod. 2009, 72, 690. (b) Tardibono, L. P.; Miller, M. J. Org. Lett. 2009, 11,1575 .

4. Committee on the review of medicines, Brit. Med. J. 1980, 910.

5. (a) Jeon, M.-K.; Kwon, J.-J.; Kim, M.-S.; Gong,Y.-D. Synlett 2008, 1651. (b) Safaei-Ghomi, J.; Hatami, A. Synth. Comm. 2008, 38, 297. (c) Cabedo, N.; Pannecoucke, X.; Quirion, J. C. Eur. J. Org. Chem. 2005, 1590. (d) Marcaccini, S.; Miliciani, M.; Pepino, R. Tetrahedron Lett. 2005, 46, 711. (e) Wiklund, P.; Rogers-Evans, M.; Bergman, J. J. Org. Chem. 2004, 69, 6371.

6. (a) Keenan, R. M.; Callahan, J. F.; Samanen, J. M.; Bondinell, W. E.; Calvo, R. R.; Chen, L.; DeBrosse, C.; Eggleston, D. S.; Haltiwanger, R. C.; Hwang, S. M.; Jakas, D. R.; Ku, T. W.; Miller, W. H.; Newlander, K. A.; Nichols, A.; Parker, M. F.; Southhall, L. S.; Uzinskas, 
I.; Vaski-Moser, J. A.; Venslavsky, W.; Wong, A. S.; Huffman, W. F. J. Med. Chem. 1999, 42, 545. (b) Knust, H.; Achermann, G.; Ballard, T.; Buettelmann, B.; Gasser, R.; Fischer, H.; Hernandez, M. C.; Knoflach,F.; Koblet, A.; Stadler, H.; Thomas, A. W.; Trube, G.; Waldmeier, P. Bioorg. Med. Chem. Lett. 2009, 19, 5940. (c) Taher, D.; Ishtaiwi, Z. N.; AlSaid, N. H. Arkivoc 2008, xvi, 154. (d) Achermann, G.; Ballard, T. M.; Blasco,F.; Broutin, P. E.; Büttelmann, B.; Fischer, H.; Graf, M.; Hernandez, M. C.; Hilty, P.; Knoflach, F.; Koblet, A.; Knust, H.; Kurt, A.; Martin, J. R.; Masciadri, R.; Porter,R. H. P.; Stadler, H.; Thomas, A. W.; Trube, G.; Wichmann, J. Bioorg. Med. Chem. Lett. 2009, 19, 5746. (e) Spencer, J.; Rathnam, R. P.; Motukuri, M.; Kotha, A. K.; Richardson, S. C. W.; Hazrati, A.; Hartley, J. A.; Male, L.; Hursthouse, M. B. J. Chem. Soc. Dalton Trans. 2009, 4299. (f) Xie, H.; Lu, C.; Yang, G.; Chen, Z. Synthesis 2009, 205.

7. Sridharan, V.; Ribelles, P.; Ramos, M. T.; Menendez, J. C. J. Org. Chem. 2009, 74, 5715.

8. Jircitano, A. J.; Sommerer, S. O.; Shelley, J. J.; Westcott, B. L. Acta Cryst. 1994, C50, 445.

9. Rossi, J-C.; Marull, M.; Larcher, N.; Taillades, J.; Pascal, R.; van der Lee, A.; Gerbie, P. Tetrahedron: Asymmetry 2008, 19, 876. 